\title{
Single and Multiple Surface Scatterings from Rough Surfaces
}

\section{Chin-Yuan Hsieh}

Department of Electronic Communication Engineering, National Kaohsiung Marine University, Kaohsiung, Taiwan 811., cyhsieh@mail.nkmu.edu.tw

\section{Shun-Hsyung Chang}

Department of Electronic Communication Engineering, National Kaohsiung Marine University, Kaohsiung, Taiwan 811.

Follow this and additional works at: https://jmstt.ntou.edu.tw/journal

Part of the Electrical and Computer Engineering Commons

\section{Recommended Citation}

Hsieh, Chin-Yuan and Chang, Shun-Hsyung (2004) "Single and Multiple Surface Scatterings from Rough Surfaces," Journal of Marine Science and Technology. Vol. 12: Iss. 4, Article 12.

DOI: $10.51400 / 2709-6998.2253$

Available at: https://jmstt.ntou.edu.tw/journal/vol12/iss4/12

This Research Article is brought to you for free and open access by Journal of Marine Science and Technology. It has been accepted for inclusion in Journal of Marine Science and Technology by an authorized editor of Journal of Marine Science and Technology. 


\title{
SINGLE AND MULTIPLE SURFACE SCATTERINGS FROM ROUGH SURFACES
}

\author{
Chin-Yuan Hsieh and Shun-Hsyung Chang
}

Key word: single scattering, multiple scattering, integral equation model.

\begin{abstract}
The objective of this paper is to study the contributions of multiple scattering and compare it with those of the single scattering from randomly rough surfaces. The existing Kirchhoff, small perturbation and IEM scattering model cannot be used to predict the multiple scattering along the azimuth angle. In this paper we modify the IEM model by adding the phase terms in Green's function and its derivative for multiple scattering. The function of phase term is significant to investigate the contribution of multiple scattering in the upward and downward directions. The contribution by multiple scattering is mainly based on the normalized surface standard deviation and the surface rms slope. We compare the model prediction with the experimental measurements for bistatic scattering along the azimuth angle. From the comparisons of model prediction with the measured data we found the contribution of multiple scattering is significant in the scattering directions of backward and forward. The origin IEM model cannot be used to predict the multiple scattering, but the modified model prediction demonstrates good agreement with the experimental data from the comparisons.
\end{abstract}

\section{INTRODUCTION}

Existing models for surface backscattering mainly focused on the surface scattering from random rough smooth surface in the plane of incidence and results were reported in various numerical, analytical and experimental studies [2, 4-8]. The existing IEM model cannot be used to predict the multiple scattering [1], therefore the study of contribution of multiple scattering from bistatic surface scattering still lacks, especially in the direction out of the specular plane. Recently further interest has arisen in bistatic surface scattering because it is possible to measure the signal from the global positioning system after it is scattered out of plane of incidence from the ocean surface. For this reason, we study the bistatic scattering trend from two-dimensional random rough surfaces out of plane of incidence in this paper.

The model developed is based upon the integral

Paper Submitted 09/21/04, Accepted 11/04/04. Author for Correspondence: Chin-Yuan Hsieh. E-mail:cyhsieh@mail.nkmu.edu.tw.

*Department of Electronic Communication Engineering, National Kaohsiung Marine University, Kaohsiung, Taiwan 811. equation method [6]. In order to account for the bistatic scattering properties out of plane of incidence, we formulate to estimate the tangential surface fields from randomly rough dielectric surfaces by a pair of integral equations. The scattering fields can be computed in terms of the tangential surface fields. Subsequently, the average scattered power and scattering coefficients can then be obtained. We evaluate these integrals and obtain the surface scattering model under various conditions.

For identifying the importance of multiple scattering we regroup the scattering terms into two parts: the single scattering and the multiple scattering. The single scattering dominates the scattering strength for scattering from moderate surface with small root mean square (rms) surface slope. Intuitively, the multiple scattering terms in the model are of a higher order compared with single scattering terms for scattering from rough surface with heights large relative to the incident wavelength, especially the cross-polarized multiple scattering. Finally, the comparisons of model predictions with the measured data are made to evaluate the model availability.

\section{MODEL DEVELOPMENT}

The governing equations for the tangential surface fields on a dielectric surface have been given by Poggio and Miller [8]. The fields in the lower medium can be written in terms of the fields in the upper medium by applying the boundary conditions on the continuity of the tangential fields. The spectral representation for the Green's function and its gradient is

$$
\begin{gathered}
G=\left(-\frac{1}{2 \pi}\right) \int \frac{j}{q} \exp \left[j u\left(x-x^{\prime}\right)+j v\left(y-y^{\prime}\right)-j q\left|z-z^{\prime}\right|\right] \\
\quad d u d v
\end{gathered}
$$

and

$$
\nabla^{\prime} G=\left(-\frac{1}{2 \pi}\right) \int \frac{\vec{g}}{q} \exp \left[j u\left(x-x^{\prime}\right)+j v\left(y-y^{\prime}\right)-j q\left|z-z^{\prime}\right|\right]
$$

$$
d u d v
$$


where $q=\sqrt{k^{2}-u^{2}-v^{2}}$ and $\vec{g}=\hat{x} u+\hat{y} v+\hat{z} q$. Without the absolute value term in the above Green's function and its derivative, the multiple scattering coefficient cannot be evaluated.

We first apply the incident field components and the surface reflectivity properties to express the tangential surface field $E_{q p}^{s}$, where $q, p$ means the incident and scatter polarization, respectively, and s means surface scattering field. The ensemble average scattered power with the given scattered field is given by

$$
\left\langle E_{q p}^{s} E_{q p}^{s^{*}}\right\rangle=\left\langle E_{q p}^{k} E_{q p}^{k^{*}}\right\rangle+2 \operatorname{Re}\left\langle E_{q p}^{c} E_{q p}^{k^{*}}\right\rangle+\left\langle E_{q p}^{c} E_{q p}^{c^{*}}\right\rangle
$$

where $R e$ means the real part operator and $*$ is the symbol for complex conjugate. To obtain the incoherent scattered power, we subtract the mean-squared power from the total power. That is,

$$
\begin{aligned}
& \left\langle E_{q p}^{s} E_{q p}^{s^{*}}\right\rangle-\left\langle E_{q p}^{s}\right\rangle\left\langle E_{q p}^{s}\right\rangle^{*} \\
& =\left\langle E_{q p}^{k} E_{q p}^{k^{*}}\right\rangle-\left\langle E_{q p}^{k}\right\rangle\left\langle E_{q p}^{k}\right\rangle^{*}+\left\langle E_{q p}^{c} E_{q p}^{c^{*}}\right\rangle-\left\langle E_{q p}^{c}\right\rangle\left\langle E_{q p}^{c}\right\rangle^{*} \\
& +2 \operatorname{Re}\left[\left\langle E_{q p}^{c} E_{q p}^{k^{*}}\right\rangle-\left\langle E_{q p}^{c}\right\rangle\left\langle E_{q p}^{k}\right\rangle^{*}\right]
\end{aligned}
$$

To carry out the ensemble average operation we need to assume the random surface distribution. Here we assume the rough surface with the properties of Gaussian distribution and define the incident and scattered wave numbers are $\vec{k}_{s}=\hat{x} k s_{x}+\hat{y} k_{s y}+\hat{z} k_{s z}$ and $\vec{k}_{i}=\hat{x} k_{x}+\hat{y} k_{y}+\hat{z} k_{z}$ separately. After the lengthy calculation of ensemble average, the average incoherent scattered power can be found sequentially.

$$
\begin{aligned}
P_{q p} & =\left|C E_{a} f_{q p}\right|^{2} A_{0} \exp \left[-\sigma^{2}\left(k_{s z}+k_{z}\right)^{2}\right] \\
& \int\left\{\exp \left[\sigma^{2}\left(k_{s z}+k_{z}\right)^{2} \rho(\xi, \varsigma)\right]-1\right\} \\
& \exp \left\{j\left(k_{s x}-k_{x}\right) \xi+j\left(k_{s y}-k_{y}\right) \varsigma\right] d \xi d \varsigma \\
& +\frac{1}{2}\left|C E_{o} /(2 \pi)\right|^{2} A_{0} \operatorname{Re}\left\{\int\left(F_{q p} f_{q p}^{*}\right)\right. \\
& \left\{k(q) k_{1}(q)\left[k_{2}(q) k_{3}(q)-1\right]\right. \\
& \left.+k(-q) k_{1}(-q)\left[k_{2}(-q) k_{3}(-q)-1\right]\right\} \\
& \exp \left[j k_{s x} \xi+j k_{s y} \varsigma+j u(\xi-\xi)+j v\left(\varsigma-\varsigma^{\prime}\right)\right. \\
& \left.-j k_{x} \xi-j k_{y} \varsigma^{\prime}\right] d \xi d \varsigma d \xi^{\prime} d \varsigma^{\prime} d u d v
\end{aligned}
$$

In (5) $\xi=x-x^{\prime \prime}, \varsigma=y-y^{\prime \prime}, \xi^{\prime}=x^{\prime}-x^{\prime \prime}, \varsigma^{\prime}=y^{\prime}-y^{\prime \prime}$, $\tau=x^{\prime \prime}-x^{\prime \prime \prime}, \kappa=y^{\prime \prime}-y^{\prime \prime \prime}, \sigma^{2}$ is the variance of the surface and $\rho\left(x-x^{\prime}, y-y^{\prime}\right)$ is the normalized surface autocorrelation function. For simplicity we refer to $\rho$ as the surface correlation.

$$
\begin{aligned}
k(q) & =\exp \left[-\sigma^{2}\left(k_{s z}^{2}+k_{z}^{2}+k_{s z} k_{z}+q^{2}-q k_{s z}+q k_{z}\right)\right] \\
k_{1}(q) & =\exp \left[-\sigma^{2}\left(k_{s z} k_{z}-q^{2}\right.\right. \\
& \left.\left.+q k_{s z}-q k_{z}\right) \rho^{1}\left(\xi-\xi^{\prime}, \varsigma-\varsigma^{\prime}\right)\right] \\
k_{2}(q) & =\exp \left[\sigma^{2}\left(k_{s z}-q\right)\left(k_{s z}+k_{z}\right) \rho^{2}(\xi, \varsigma)\right] \\
k_{3}(q) & =\exp \left[\sigma^{2}\left(k_{z}+q\right)\left(k_{s z}+k_{z}\right) \rho^{3}\left(\xi^{\prime}, \varsigma^{\prime}\right)\right]
\end{aligned}
$$

and $A_{o}$ is the illuminated area.

The bistatic scattering coefficient is related to the ensemble average scattered power expression as

$$
\sigma_{q p}^{0}=\left(4 \pi R^{2} P_{q p}\right) /\left(E_{0}^{2} A_{0}\right)
$$

Therefore the bistatic scattering coefficient can then be expressed as

$$
\begin{aligned}
\sigma_{q p}^{o}(s)= & \frac{k^{2}}{4 \pi}\left|f_{q p}\right|^{2} e^{-\sigma^{2}\left(k_{s z^{+}}+k_{z}\right)^{2}} \cdot \int\left[e^{\sigma^{2}\left(k_{s z}+k_{z}\right)^{2} \rho(\xi, \varsigma)}-1\right] \\
& \cdot e^{j\left(k_{s x}-k_{x}\right) \xi+j\left(k_{s y}-k_{y}\right) \varsigma} d \xi d \varsigma \\
& +\frac{k^{2}}{32 \pi^{3}} \operatorname{Re}\left\{\int\left[f_{q p}^{*} F_{q p}\left(-k_{x},-k_{y}\right)\right]\right. \\
& \cdot \iint\left\{k(q) k_{1}(q)\left[k_{2}(q) k_{3}(q)-1\right]\right. \\
& \left.+k(q) k_{1}(q)\left[k_{2}(q) k_{3}(q)-1\right]\right\} \\
& \cdot e^{j k_{s x} \xi+j k_{s y} \varsigma+j u\left(\xi-\xi^{\prime}\right)+j v\left(\varsigma-\varsigma^{\prime}\right)-j k_{x} \xi^{\prime}-j k_{y} \xi^{\prime}} \\
& \left.d \xi d \varsigma d \xi^{\prime} d \varsigma^{\prime} d u d v\right\}
\end{aligned}
$$

The above equations give the complete representation of the bistatic scattering coefficient for a randomly rough surface. The scattered field coefficients $f_{q p}$ and $F_{q p}$ are given in appendix of $[7,8]$.

\section{MODEL PREDICTION}

A set of plot of the cross- and like-polarized bistatic scattering coefficient versus the azimuth angle is shown in Figures 1 through 2, respectively. In the figures zero value of the azimuth angle corresponds to the forward scattering and $180^{\circ}$ of azimuth angle represents the backscatter direction in the incident plane. However $90^{\circ}$ represents the direction orthogonal to the plane of incidence.

Figures 1 and 2 are the azimuth slice of the bistatic 


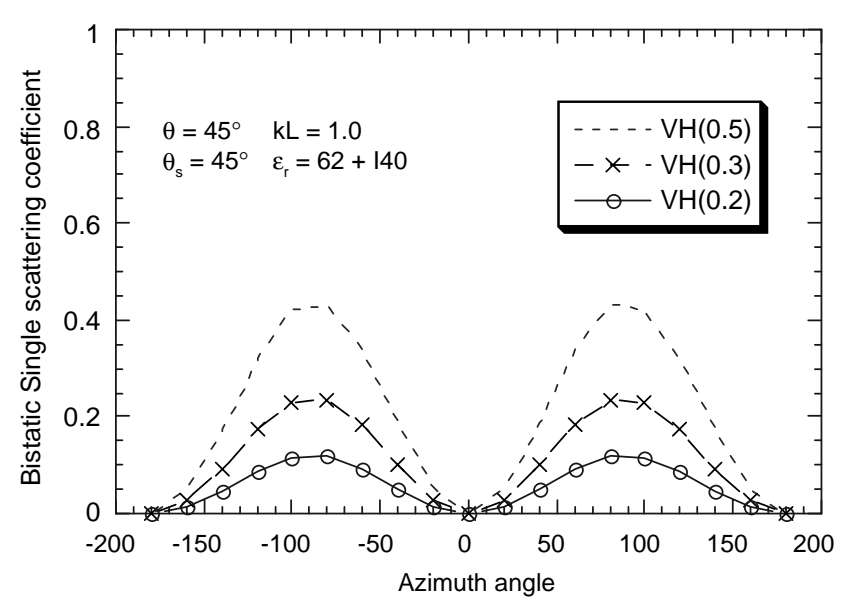

Fig. 1. Comparisons of cross VH-polarized single scattering from rough surfaces with different normalized surface rms height along the azimuth angle.

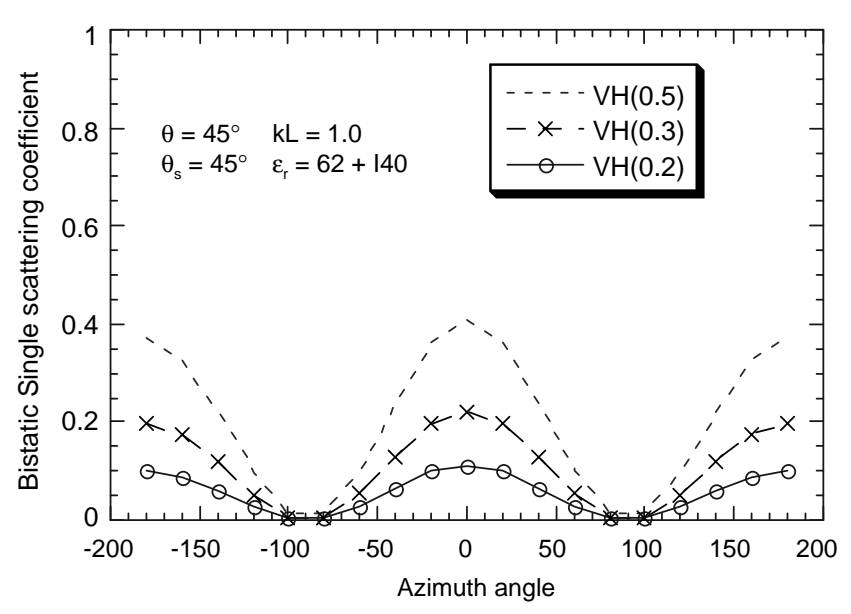

Fig. 2. Comparisons of like HH-polarized single scattering from rough surfaces with different normalized surface rms height along the azimuth angle.

scattering pattern. The incidence angle is $45^{\circ}$ and the scattering angle is also $45^{\circ}$. The normalized surface height to the incidence wave numbers are $0.2,0.3$ and 0.5 , respectively and normalized surface correlation length is 1.0. The rms surface slopes are $0.2828,0.4242$ and 0.707 , respectively. The surface of water-soaked form bricks has complex dielectric constant $62+i 40$. We make the comparisons of like- and cross-polarized scattering from rough surface with different rms slope in figures. The azimuth pattern exhibits a strong lobe along the specular direction for the like-polarization components. For cross polarization the scattering component exhibits a local null along the specular direction in Figure 1. On the contrary, the azimuth pattern exhibits a strong lobe along the orthogonal plane of

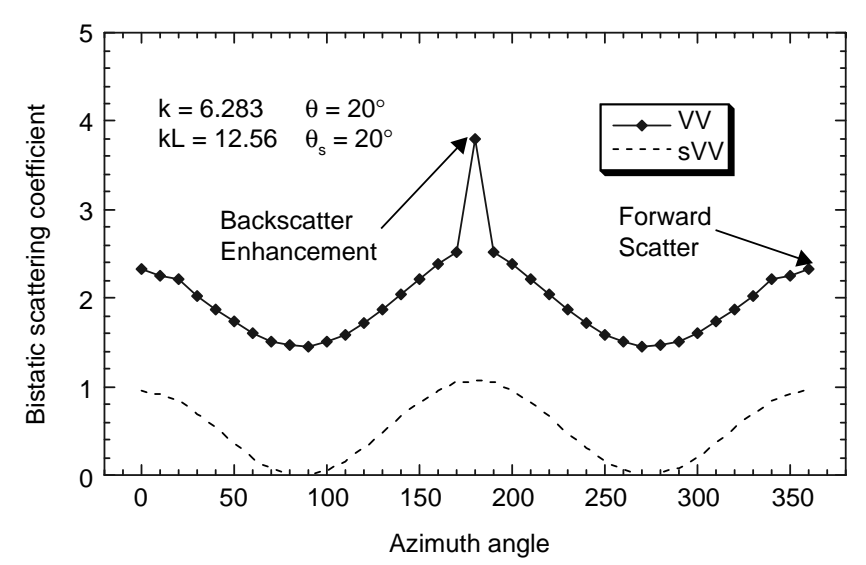

Fig. 3. Comparisons of single with total like VV-polarized scattering from rough surfaces along the azimuth angle.

incidence for the cross-polarization components. For the like polarization the like-polarized scattering component exhibits a local null along the plane orthogonal to the plane of incidence and has a strong lobe along the direction of incident plane in Figure 2. Those satisfy the scattering properties that the single scattering makes a major contribution along the specular direction and multiple scattering makes it along the plane orthogonal to the plane of incidence. For surfaces with moderate surface height and rms slope the single scattering along the specular direction is important in like polarized and multiple scattering contributions becomes negligible. For surfaces with moderate surface height and rms slope the single scattering along the specular direction is important in like polarized and multiple scattering contributions becomes negligible [3]. Multiple scattering is the major source for cross polarization along the plane orthogonal to the plane of incidence. The figures reveal that the electromagnetic waves scattered out of the plane of incidence are strongly dependent on the polarization orientation of the incident electric field.

Figures 3 and 4 are the azimuth slice of the bistatic scattering pattern. The incidence angle is $20^{\circ}$ and the scattering angle is also $20^{\circ}$. The normalized surface height to the incidence wave number is 6.285 and normalized surface correlation length is 12.56 . The rms surface slope is 0.707 . The backscatter enhancement occurs due to the contribution of multiple scattering in Figures 3 and 4 . The single scattering has no contribution to the backscatter enhancement, because the phenomenon of backscatter enhancement is caused by the phase terms of the Green's function in a pair of integral equations. The phase terms of the Green's function are the major factor for multiple scattering.

For VV-polarized scattering the multiple scattering keeps constant along the azimuth angle except the 


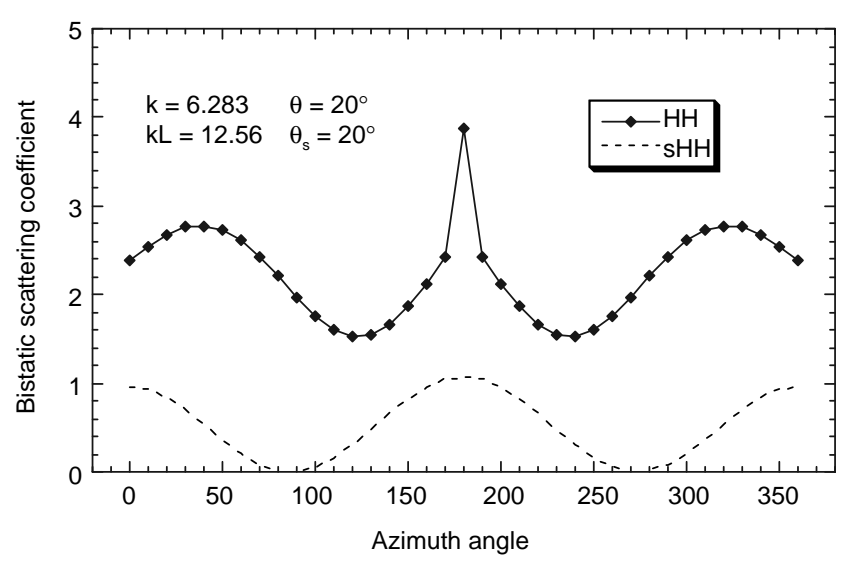

Fig. 4. Comparisons of single with total like HH-polarized bistatic scattering from rough surfaces along the azimuth angle.

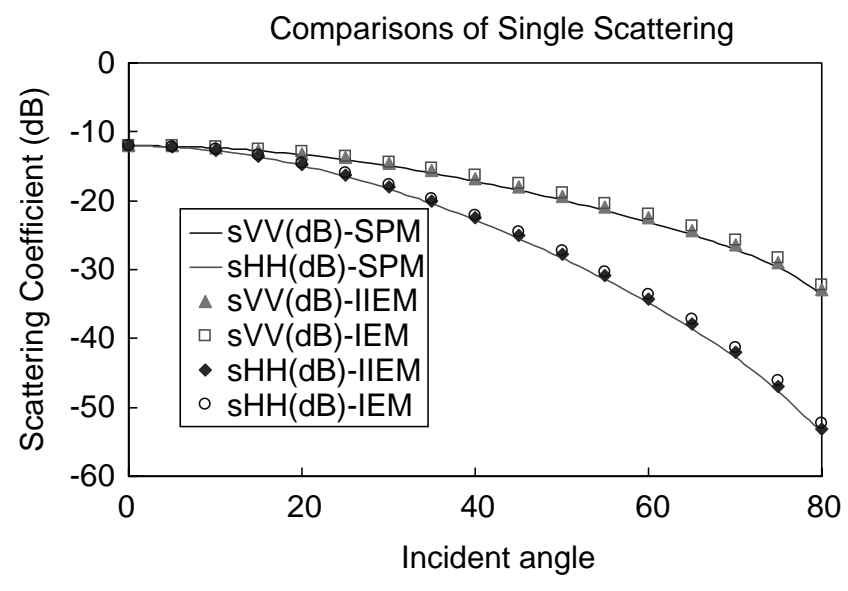

Fig. 5. Comparisons of SPM, IIEM, and IEM for like-polarized single scattering.

behavior of backscatter enhancement in the backward scattering direction along the incident plane. However, for HH-polarized scattering the multiple scattering, total scattering minus the single scattering, fluctuates along the azimuth angle due to the Brewster angle.

\section{COMPARISONS}

First we compare the single like-polarized backscattering strength of IEM model with those of IIEM and small perturbation model (SPM) to evaluate the scattering behavior from the slightly rough surface with normalized surface height 0.1 , correlation length 1.0 and relative dielectric constant 20. From Figure 5 we found the difference among them is less than $1 \mathrm{~dB}$. Further we also compare the single like-polarized scattering characteristics of Kirchhoff Model (KM) with those of IIEM and IEM from the rough surface with

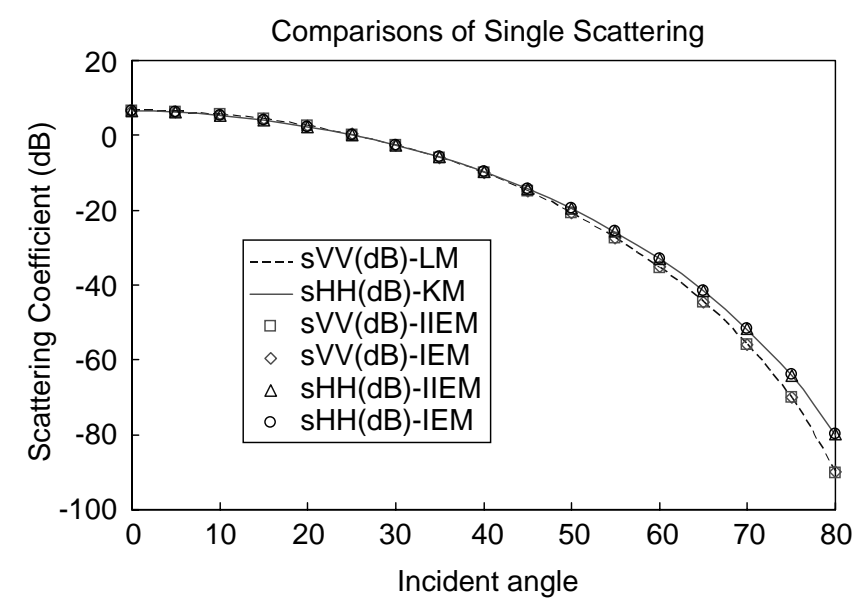

Fig. 6. Comparisons of KM, IIEM, and IEM for like-polarized single scattering.

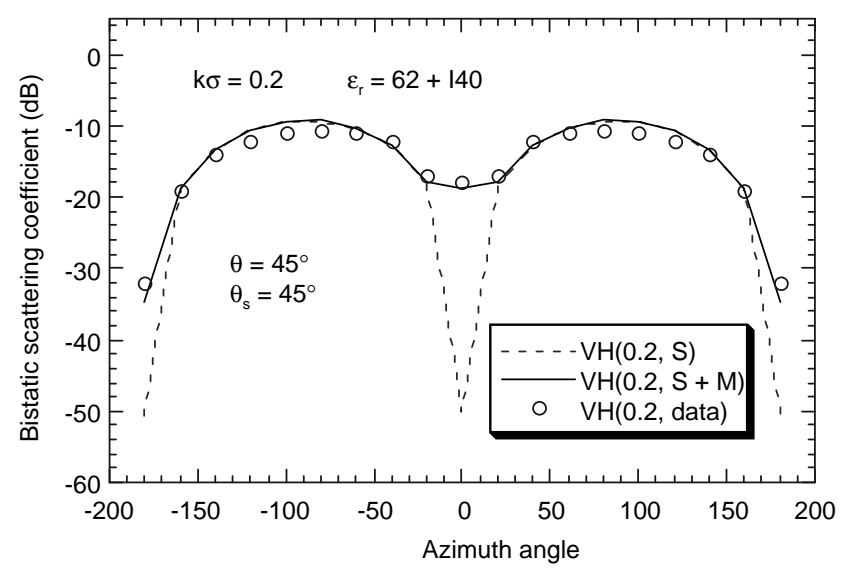

Fig. 7. The comparisons of cross-polarized model prediction contribution with the measured data along azimuth angle from random rough surfaces. $S$ means the single scattering and $S+$ M means total scattering strength.

normalized surface height 1.5 , correlation length 8.4 and relative dielectric constant 81 along the incidence plane. From the comparisons in Figure 6 we also found the differences among them are less than $1 \mathrm{~dB}$. Form the comparisons we conclude the IEM model may be the bridge of SPM and KM models between the high and low frequency band.

For evaluating the ensemble scatter averages the multiple scattering cannot be ignored. Furthermore, backscattering enhancement mainly comes from the multiple surface scattering. The comparisons of crosspolarized single and multiple scattering from random rough surfaces along azimuth angle were made in Figure 7. In Figure 7 we also compare the model prediction with the measured data for bistatic scattering at fixed incident and scattered angles along the azimuth 
angle from rough surfaces. The bistatic scatter was measured from a water-soaked form bricks surface with complex dielectric constant $62+i 40$, normalized surface height 0.2 and correlation length 1.0. These measured data provide a test for the model prediction.

From the comparisons the contribution of crosspolarized single scattering is significant in the scattering directions of incident plane, however the multiple scattering has the significant contribution in the scattering directions of backward and forward. The crosspolarized scattering has no contribution in the plane of incidence. Figure 7 shows the contribution of multiple scattering from rough surfaces with rms slope less than 0.2 can be ignored out of plane of incidence. The main contribution of cross-polarized scattering out of plane of incidence comes from the single scattering for the smooth rough surface. The scattering behavior complies with the electromagnetic wave scattering property. The contributions by multiple scattering are based on the normalized surface standard deviation and the surface rms slope. From the comparisons the excellent agreement between the measured data made by Hauck et al. [2] and model prediction reach for the cross polarization along the azimuth direction. The difference between the measured data and total scattering strength is within $1 \mathrm{~dB}$. Without the contribution of multiple scattering the difference between the measured data and model prediction cannot be satisfied in the plane of incidence.

\section{REFERENCES}

1. Fung, A.K., Microwave Scattering and Emission Models and Their Applications, Artech House, Boston
(1994).

2. Hauck, B., Ulaby, F., and DeRoo, R., "Polarimetric Bistatic-Measurement Facility for Point and Distributed Targets," IEEE Antenna Propagat. Mag., Vol. 40, No. 1, pp. 31-41 (1998).

3. Hsieh, C.Y., Fung, A.K., Nesti, G., Sieber, A., and Coppo, P., "A Further Study of the IEM Surface Scattering Model," IEEE Trans. Geosci. Remote Sens., Vol. 35, No. 4, pp. 901-909 (1997).

4. Hsieh, C.Y. and Fung, A.K., "Application of an Extended IEM to Multiple Surface Scattering and Backscatter Enhancement," J. Electromagn. Waves Appl. Prog. Electromagn. Res., Vol. 13, pp. 121-135 (1999).

5. Ishimaru, A. and Chen, J.S., "Scattering from Very Rough Surfaces Based on the Modified Second-Order Kirchhoff Approximation with Angular and Propagation Shadowing," JASA, Vol. 88, pp. 1877-1883 (1990).

6. Kim, M.J., Dainty, J.C., Friberg, A.T., and A.J. Sant, "Experimental Study of Enhanced Backscattering from One- and Two-Dimensional Random Rough Surfaces," J. Opt. Soc. Am. A, Vol. 7, pp. 569-577 (1990).

7. Maradudin, A., Michel, T., McGurn, A.R., and Mendez, E.R., "Enhanced Backscattering of Light from a Random Grating," Annals Physics, Vol. 203, pp. 255307 (1990).

8. Poggio, A.J. and E.K. Miller, "Integral Equation Solution of Three Dimensional Scattering Problems," Computer Techniques for Electromagnetics, Pergamon, New York, Chapter 4 (1973).

9. Soto-Crespo, J.M. and Nieto-Vesperinas, M., "Electromagnetic Scattering from very Rough Random Surfaces and Deep Reflection Gratings," J. Opt. Soc. Am. A, Vol. 6, pp. 367-384 (1989). 